\title{
Inhibition of return spreads across 3-D space
}

\author{
JAN THEEUWES \\ Vrije Universiteit, Amsterdam, The Netherlands \\ and \\ JAY PRATT \\ University of Toronto, Toronto, Canada
}

\begin{abstract}
Focusing attention to a location in 3-D space operates much the same as in 2-D space. Attending a location in 2-D space is followed by a selective inhibitory aftereffect known as inhibition of return (IOR). Here, we report the results of two 3-D reflexive cuing studies in which depth was defined by binocular disparity. As has been shown before, attentional cuing was specific for $x-y-z$ locations. However, the present results show that IOR is not depth specific. After a specific location in $x-y-z$ is cued, IOR occurs for the depth plane in front of and behind the cued location. The finding that IOR spreads across depth planes may be related to how inhibited locations are encoded in the superior colliculus. We argue that the functional role of a depth-blind IOR is to bias attention against going back to any part of a previously attended object.
\end{abstract}

In order to behave in a goal-directed manner, it is important that we orient to relevant events in the visual world. When attention is oriented to a peripheral visual event (e.g., an abrupt onset), there is first facilitation of processing of nearby stimuli. This facilitatory effect, called attentional cuing, is due to a reflexive orienting of attention toward the source of stimulation (e.g., Posner, 1980). Following this brief period of facilitation, there is a long-lasting inhibitory effect in which there is delayed responding to stimuli presented at or near the subsequently cued location. This effect, termed inhibition of return (IOR; Posner \& Cohen, 1984; see Klein, 2000, and Taylor \& Klein, 1998, for reviews), usually begins approximately $300 \mathrm{msec}$ after the presentation of the peripheral event and lasts as long as 4,000 msec (see, e.g., Tassinari \& Berlucchi, 1995). By biasing attention away from recently attended locations, our orienting behavior is made more efficient.

The efficiency of attentional orienting not only holds in 2-D space, but also occurs in 3-D space. Several studies provide converging evidence that the visual system can focus attention to a particular location in depth defined by binocular disparity (Atchley, Kramer, Andersen, \& Theeuwes, 1997; Hoffman \& Mueller, 1994; Nakayama \& Silverman, 1986; Theeuwes, Atchley, \& Kramer, 1998). For example, Andersen (1990) and Andersen and Kramer (1993), employing an Eriksen flanker task, showed that the effect of

We thank Bruce Milliken, Adrian von Mühlenen, Mieke Donk, and John Wixted for their excellent comments and suggestions on an earlier draft of this paper and Patrick van Olffen and Donny Chen for their assistance in conducting the studies. This research was supported by an NSERC grant to J. Pratt. Correspondence concerning this article should be addressed to J. Theeuwes, Department of Cognitive Psychology, Vrije Universiteit, Van Der Boechorststraat 1, 1081 BT Amsterdam, The Netherlands (e-mail: j.theeuwes@ psy.vu.nl). incompatible flankers diminished as they were moved away from the target in depth, which is comparable to the effect of increasing retinal distance in 2-D (cf. Eriksen \& Eriksen, 1974). Hoffman and Mueller (1994), who also used binocular disparity to induce depth, showed that the brightening of a cue can capture attention and produce attentional cuing effects to a 3-D location (i.e., $x-y-z$ coordinates) in a way similar to that which has been shown in 2-D space (see, e.g., Theeuwes, 1991). Overall, there is considerable evidence that attention operates in 3-D much as in 2-D.

Even though it has been demonstrated that attention can be oriented to a specific location in depth, it remains unclear whether the facilitation of processing information at a particular location in depth is followed by a subsequent inhibition at a particular location in 3-D space. If orienting in depth operates as efficiently as in 2-D, then one would expect facilitation of processing at a particular location in depth to be followed by subsequent inhibition at that location.

\section{EXPERIMENT 1}

Experiment 1 was conducted to establish reflexive attentional orienting in 3-D. We used a variant to the classic Posner (1980) cuing task presented in 3-D (i.e., two boxes in the front plane and two in the back plane). The cue consisted of the brightening of one randomly selected premask. In order to assess cuing effects for $x-y$ space independently of effects in $z$ (depth), we used a paradigm in which elements in the front depth plane were basically at the same $x-y$ coordinates as elements in the back depth plane (see also Hoffman and Mueller, 1994).

\section{Method}

The trial sequence is shown in Figure 1. The fixation display consisted of a central cross with four gray figure- 8 premasks $\left(1.3^{\circ} \times\right.$ 
$2.5^{\circ}$ ) presented at $4.4^{\circ}$ to the left and right sides. The figure- $8 \mathrm{~s}$ in the back plane were slightly displaced laterally, by $0.4^{\circ}$ horizontally and $0.3^{\circ}$ vertically. All premasks were presented in gray. To induce a strong perception of depth across the two depth planes, small (less than $0.1^{\circ}$ ) gray dots were presented at random locations in the front and back planes. The dots appeared everywhere except at the locations of the premasks. In the 3-D condition, two figure- 8 premasks were presented in the front plane (one to the right and one to the left), and two figure- 8 premasks were presented in the back plane (one to the right and one to the left). The binocular disparity between the depth planes was $\pm 15^{\prime}$ of arc relative to the fusion display at which the fixation cross was presented. The 2-D condition was identical to the 3-D condition except that all four figure-8s appeared at zero disparity- that is, at the same plane as the fixation point. Binocular fusion of separate left and right eyes was achieved by crystal shutter glasses (Stereographics Corp.) that were synchronized with alternating frames $(60 \mathrm{~Hz}$ per eye).

In both the 3-D and 2-D conditions, the participants wore the crystal shutter glasses. At the beginning of a trial, the participants fixated the center fixation cross. After $1,200 \mathrm{msec}$, one of the figure- 8 premasks brightened for $33 \mathrm{msec}$. Following a $50-\mathrm{msec}$ interstimulus interval (ISI), the target was presented by removing segments of one of the figure- $8 \mathrm{~s}$ to produce either an $\mathrm{H}$ or an $\mathrm{S}$. The target was presented for $116 \mathrm{msec}$. The display went blank until the next trial began. There was no relationship between the location of the cue and the location of the target (i.e., cue validity was $25 \%$ ). The time from cue onset to display offset was $200 \mathrm{msec}$, a time frame that is considered to be too short to initiate vergence eye movements (Rashbass $\&$ Westheimer, 1961). During a trial, the participants were instructed not to make eye movements and to press the "Z" key for an $\mathrm{H}$ target and the "?" key for an S target as quickly as possible. Each participant performed in both the 2-D and the 3-D conditions, and the order was balanced across participants. Before each 2-D or 3-D condition, the participants received 80 practice trials followed by 160 experimental trials. Within a block of trials, there was an equal number of trials combining the following factors: cue location (4), target location (4), and target (S vs. H). These trials were presented in a random order, creating four conditions: (1) cue and target at the same loca- tion and depth (valid condition), (2) cue on one side of the visual field and target on the other side, but at the same depth plane (invalid in $x-y$ ), (3) cue in one depth plane and target in other depth plane, but basically at the same $x-y$ coordinates (invalid in $z$ ), and (4) cue on one side and target on the other side, both in different depth planes (invalid in $x-y$ and $z$ ).

The participants had to pass a screening test to determine whether they were able to perceive depth on the basis of binocular disparity (see Atchley \& Kramer, 2001, for a description of the screening test). Participants who committed more than $25 \%$ errors in the preliminary practice sessions were excluded. In total, 10 young adults ranging in age from 19 to 28 years were paid for their participation in the study.

\section{Results and Discussion}

Reaction times (RTs) longer than $1,000 \mathrm{msec}$ were counted as errors and accounted for a loss of $2.4 \%$ of the trials. Mean RTs and errors were entered into separate analyses of variance (ANOVAs) with depth (3-D vs. 2-D) and validity (valid vs. invalid in $x-y$ vs. invalid in $z$ vs. invalid in $x-y$ and $z$ ) as factors. There were main effects of depth $[F(1,9)=7.3, p<.05]$ and of validity $[F(3,27)=25.5$, $p<.001]$. The interaction was also reliable $[F(3,27)=$ $3.5, p<.05]$. Table 1 gives an overview of the results.

Additional planned comparisons showed that RTs were shorter for the valid condition than for the invalid-in- $x-y$ condition for both the 3-D (594 vs. $646 \mathrm{msec}, p<.01$ ) and 2 -D conditions ( 580 vs. $610 \mathrm{msec}, p<.05$ ). When the target appeared at the same $x-y$ location (invalid-in- $z$ condition), RTs were significantly longer when the target and cue appeared in different depth planes (3-D; 594 vs. $640 \mathrm{msec}, p<.01)$ but not when they appeared in the same depth plane (2-D; 580 vs. $593 \mathrm{msec}, p=.18$ ). These findings suggest that the brightening of the cue produced

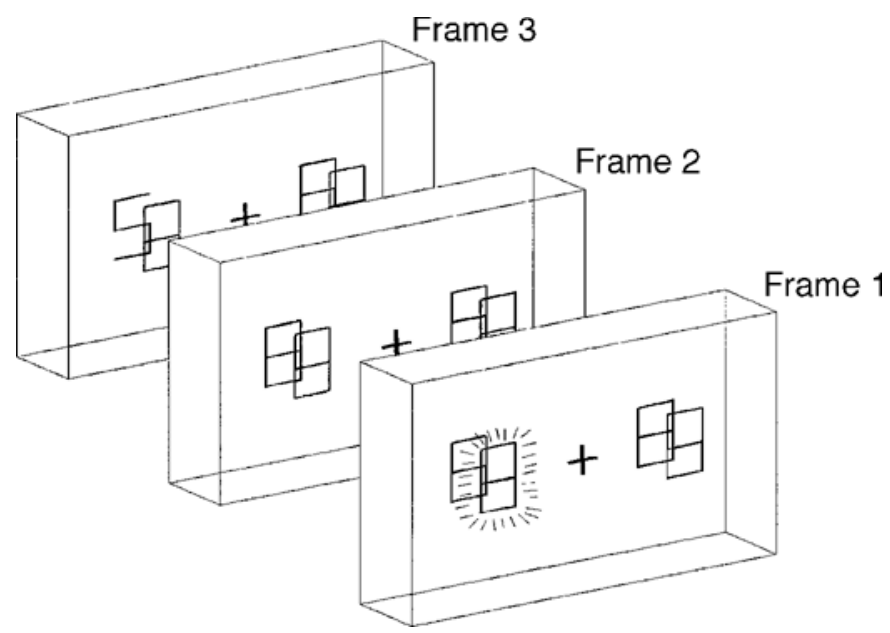

Figure 1. Sequence of frames on a given trial in the 3-D condition in Experiment 1. After $1,200 \mathrm{msec}$, one of the premasks was brightened for $33 \mathrm{msec}$ (Frame 1). After an interstimulus interval of $50 \mathrm{msec}$ (Frame 2), the target (an $S$ or an $H$ ) was revealed by a switch of the segments of the figure-8 premask (Frame 3). The target display was presented for $116 \mathrm{msec}$. In this example, the cue is presented in the front depth plane and the target (the letter $S$ ) is presented in the back depth plane (the invalid depth condition). The same basic sequence was used in Experiment 2, with the duration of Frame 2 being increased to $850 \mathrm{msec}$. 
Table 1

Experiment 1: Mean Correct Reaction Times (RTs, in Milliseconds) and Percentage of Errors (\% Error) for Target Discriminations in the 3-D and 2-D Conditions

\begin{tabular}{llcllr}
\hline & \multicolumn{2}{c}{ 3-D } & & \multicolumn{2}{c}{ 2-D } \\
\cline { 2 - 3 } \cline { 6 - 6 } \multicolumn{1}{c}{ Condition } & RT & \% Error & & RT & \% Error \\
\hline Valid & 594 & 9.5 & & 580 & 8.0 \\
Invalid in $x-y$ & 646 & 10.8 & & 610 & 12.8 \\
Invalid in $z$ & 640 & 15.8 & & 593 & 8.8 \\
Invalid in $x-y$ and $z$ & 652 & 10.0 & & 618 & 8.8 \\
\hline
\end{tabular}

an automatic shift of attention to its $x-y-z$ location. The time to shift from one depth plane to another was about $46 \mathrm{msec}$, an effect similar to that reported by Hoffman and Mueller (1994), who employed a similar paradigm.

In order to determine whether there were asymmetry effects in attending to the front or to the back plane, a separate cue (front plane vs. back plane) $\times$ target (front plane vs. back plane) $\times x-y$ validity ( $x-y$ valid vs. $x-y$ invalid) ANOVA was conducted on the data from the 3-D condition. There was a main effect of $x$ - $y$ validity $[F(1,9)=$ $27.9, p<.001]$, and the interaction between cue and target was reliable $[F(1,9)=22.5, p<.01]$. More importantly, the three-way interaction was significant $[F(1,9)=$ $21.3, p<.002]$. As can be seen in Figure 2, the cuing effect was largely restricted to the $x-y-z$ location that was cued. In other words, the shortest RTs were obtained when both cue and target were in the front plane at a valid $x-y$ location or when both were in the back plane at a valid $x-y$ location. The $x-y$-valid condition represents the condition in which the cue and target were presented at the same $x-y$ location. The difference between the conditions target in front and target in back for the valid $x$-y location represents the cuing-in-depth effect.

As is clear from Figure 2, attending to the front plane (left panel) when the target appeared in the back plane produced a 52-msec cuing-in-deptheffect. Attending to the back plane (right panel) when the target appeared in the front plane produced a cuing-in-depth effect of $40 \mathrm{msec}$. This difference in depth cuing was not reliable $(F=0.27)$, indicating that there was no asymmetry effect in attending to the front or to the back. It is worth noting that asymmetry effects have been reported in some studies (e.g., Andersen, 1990; Andersen \& Kramer, 1993) but not in others (e.g., Theeuwes et al., 1998). Currently, there is no clear evidence as to what the mitigating factor might be, but our results clearly indicate no asymmetry in orienting attention between depth planes at a short SOA. There was a depth $\times$ validity interaction on errors $[F(3,27)=4.4, p<.05]$. Additional planned comparisons showed that, in accordance with the RT data, more errors were made in the invalid- $z$ condition than in the valid condition for the 3-D depth condition.

\section{EXPERIMENT 2}

Since Experiment 1 established the typical reflexive attentional cuing in 3-D in a task that allowed independent assessment of $x-y$ and depth cuing effects, it was now possible to determine IOR in 3-D. In the present experiment, we used the same basic methods as in Experiment 1, but the ISI was increased from 50 to $850 \mathrm{msec}$ (in the typical range for many IOR experiments, e.g., Bennett \& Pratt, 2001). Moreover, IOR has been reported for 2-D letter discrimination tasks (see, e.g., Lupiáñez, Milliken, Solano,
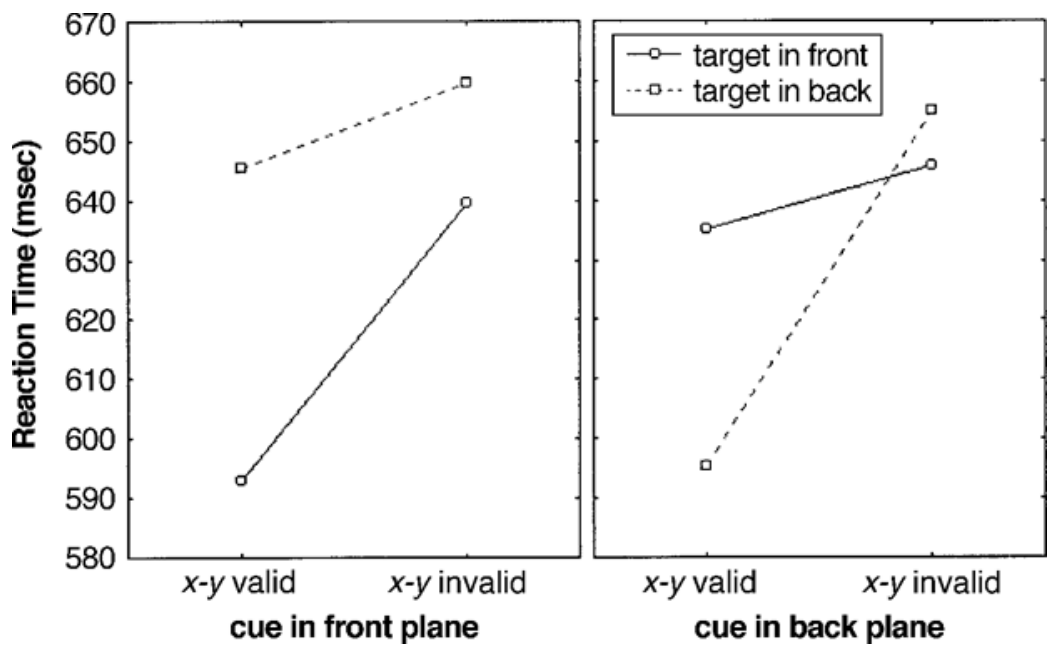

Figure 2. Experiment 1: Mean reaction times (RTs) for $x$-y cue validity in 3-D for conditions in which the cue was either in the front plane or in the back plane and the target was either in the front plane or in the back plane. Note that the cuing effect was largely restricted to the $x-y-z$ location that was cued. The fastest RTs were obtained in conditions in which both cue and target were in the front plane (left panel) or both were in the back plane (right panel) and valid in $x-y$ space. The difference between the target-in-front and target-in-back conditions for the valid $x-y$ location represents the cuing-in-depth effect. 
Table 2

Experiment 2: Mean Correct Reaction Times (RTs, in Milliseconds) and Percentage of Errors (\% Error) for Target Discriminations in the 3-D and 2-D Conditions

\begin{tabular}{lccccc}
\hline & \multicolumn{2}{c}{ 3-D } & & \multicolumn{2}{c}{ 2-D } \\
\cline { 2 - 3 } \cline { 5 - 6 } \multicolumn{1}{c}{ Condition } & RT & \% Error & & RT & \% Error \\
\hline Valid & 558 & 6.7 & & 544 & 4.5 \\
Invalid in $x-y$ & 546 & 7.4 & & 531 & 5.5 \\
Invalid in $z$ & 559 & 8.1 & & 550 & 4.5 \\
Invalid in $x-y$ and $z$ & 548 & 7.2 & & 534 & 5.7 \\
\hline
\end{tabular}

Weaver, \& Tipper, 2001; Pratt \& Abrams, 1999) similar to the task used in the present experiment.

\section{Method}

The task was the same as the one used in Experiment 1 except that the ISI between the presentation of the cue and that of the target was $850 \mathrm{msec}$. In order to ensure that attention was oriented back to the center after peripheral cuing, the fixation cross was switched off $600 \mathrm{msec}$ before cue presentation and switched on $250 \mathrm{msec}$ after cue presentation. There were 24 participants, ranging in age from 19 to 30 years.

\section{Results}

RTs longer than $1,000 \mathrm{msec}$ were counted as errors and accounted for a loss of $1.2 \%$ of the trials. A depth $\times$ validity ANOVA revealed a main effect for validity only $[F(3,69)=8.4, p<.01]$. Table 2 gives an overview of the results. Additional planned comparisons showed that when the target appeared at the cued location (valid condition), the participants were significantly slower than when it appeared on the other side of the visual field (invalid in $x-y$ ) both for the 3-D condition (558 vs. $546 \mathrm{msec}, p<.05$ ) and for the 2-D condition (544 vs. $531 \mathrm{msec}, p<.05$ ), indicating the presence of the classic IOR effect in 2-D. To determine whether there was a specific 3-D IOR effect, for the 3-D conditions we compared the valid condition with the invalid-in- $z$ condition. These conditions did not differ (valid $=558 \mathrm{msec}$, invalid in $z=559 \mathrm{msec}, F<1$ ), suggesting that the inhibition was just as strong at the cued location as at the same location in $x-y$ but at a different depth.

To determine whether the IOR effect for depth was asymmetric, a separate cue (front plane vs. back plane) $\times$ target (front plane vs. back plane) $\times x-y$ validity $(x-y$ valid vs. $x-y$ invalid) ANOVA was conducted on the data from the 3-D condition. There was a main effect of target $[F(1,23)=24.4, p<.0001]$, with responses to targets in the front plane being faster than responses to targets in the back plane. This is because the front plane placeholders partially occlude the back plane targets, making the identification of the back plane targets more difficult. There was also a main effect of $x-y$ validity, with responses to targets at valid locations in $x-y$ space being slower than those to targets at invalid $x-y$ locations $[F(1,23)=6.9$, $p<.05]$ - the previously noted IOR effect. Importantly, the factor of cue (front plane vs. back plane) did not interact with either target or validity, nor was there a three-way interaction $\left(F_{\mathrm{S}}<1\right)$. This indicates that the 3-D IOR effect was symmetric: The same amount of IOR was produced in the invalid depth plane for both front and back cues. This symmetric IOR effect for depth can be seen in Figure 3.

There was a main effect of depth (2-D vs. 3-D) on errors $[F(1,23)=10.6, p<.01]$, indicating that the participants made more errors in the 3 -D condition (7.4\%) than in the 2-D condition $(5.0 \%)$.

\section{CONCLUSIONS}

The present results indicate that both the facilitatory and inhibitory consequences of orienting attention to a specific location are not limited to two spatial dimensions but occur in 3-D space. Experiment 1 provides further evidence that attentional cuing effects are found for specific

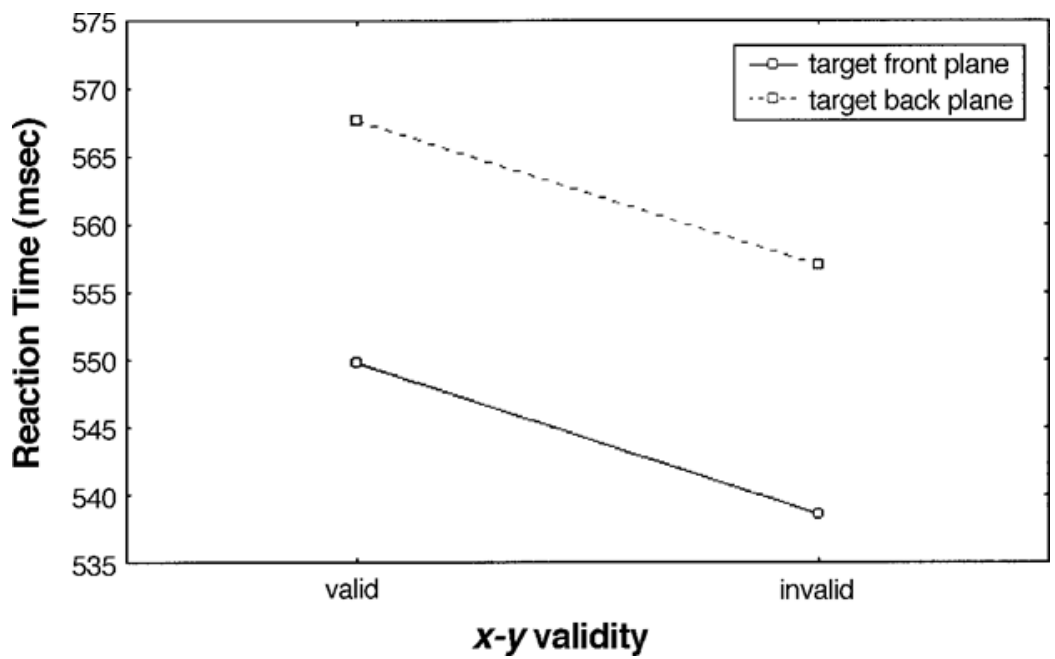

Figure 3. Experiment 2: Mean reaction times for $x-y$ cue validity in 3-D for targets in the front plane and targets in the back plane. Note that the IOR effect was not mitigated by cuing in depth. 
$x-y-z$ locations, a result that converges with the findings of several other studies (Atchley et al., 1997; Hoffman \& Mueller, 1994; Nakayama \& Silverman, 1986; Theeuwes et al., 1998). Furthermore, the symmetrical cuing effect (i.e., no differences between front and back cues) is consistent with Theeuwes et al. (1998).

More importantly, the present results also indicate that IOR occurs in 3-D space. Specifically, if a location in $x-y$ space is inhibited, the depth plane in front of and behind the cued location is inhibited as well. In other words, IOR appears to spread not only in $x-y$ space (see, e.g., Bennett $\&$ Pratt, 2001) but also in depth. The finding that IOR is not limited to the previously cued depth plane has implications for how attention is allocated in visual space as a consequence of a peripheral cue. One such implication is that these results add to a growing set of findings that suggest that later IOR effects are not simply mirror images of earlier cuing effects. For example, Pratt, Hillis, and Gold (2001) have shown that cues of different physical properties produce a variety of cuing effects (ranging from weakly inhibitory to strongly facilitatory), whereas all produce reliable IOR effects.

Another implication regards how cuing effects and IOR are generated. There is considerable evidence that the abrupt onset of a peripheral object (i.e., a cue) results in the programming of a saccadic eye movement to the corresponding location. Presumably, such an eye movement is programmed to a specific $x-y-z$ location in space (since eye movements are typically thought to be programmed in environmental coordinates; see Sparks \& Mays, 1990). There is also considerable evidence that attention is oriented to the saccade location before the saccade is initiated or if the saccade is cancelled (e.g., Deubel \& Schneider, 1996; Godijn \& Theeuwes, 2002; Rafal, Calabresi, Brennan, \& Sciolto, 1989). Thus, it is likely that this movement of attention is also programmed in $x-y-z$ space, and this results in the attentional cuing effects at a specific location and at a specific depth. However, the present study shows that attentional cuing to a location in 3-D space is not followed by a depth-specific inhibitory aftereffect. Rather, IOR spreads across depth planes. One speculation as to why IOR is not depth specific may be related to the makeup of the superior colliculus (SC), a midbrain oculomotor structure that plays an important role in IOR (Klein, 2000). Some recent evidence suggests that the superior colliculus has a topographic map of visual space that codes in retinal coordinates (Klier, Wang, \& Crawford, 2001). This would imply that the SC is basically depth blind (i.e., it does not code depth), and this could be the reason why IOR spreads across 3-D space.

From a functional point of view, it is useful that IOR spreads in depth. The function of IOR is to bias orienting away from recently inspected locations, thereby facilitating visual search (see, e.g., Klein, 2000). When we search an object, it is useful that once we attend one portion of the object (e.g., the front), IOR biases attention from going back to any part of the object. A depth-blind inhibitory effect that spreads over $x-y-z$ space would provide this functional foraging capability.

\section{REFERENCES}

Andersen, G. J. (1990). Focused attention in three-dimensional space. Perception \& Psychopysics, 47, 112-120.

Andersen, G. J., \& Kramer, A. F. (1993). Limits of focused attention in three-dimensional space. Perception \& Psychophysics, 53, 658-667.

Atchley, P., \& Kramer, A. F. (2001). Object- and space-based attentional selection in three-dimensional space. Visual Cognition, 8, 1-32.

Atchley, P., Kramer, A. F., Andersen, G. J., \& Theeuwes, J. (1997). Spatial cuing in a stereoscopic display: Evidence for a "depth-aware" attentional focus. Psychonomic Bulletin \& Review, 4, 524-529.

Bennett, P. J., \& Pratt, J. (2001). The spatial distribution of inhibition of return. Psychological Science, 12, 76-81.

Deubel, H., \& Schneider, W. X. (1996). Saccade target selection and object recognition: Evidence for a common attentional mechanism. Vision Research, 6, 1827-1837.

ERIKSEN, B. A., \& ERIKSEN, C. W. (1974). Effects of noise letters upon the identification of a target letter in a nonsearch task. Perception \& Psychophysics, 16, 143-149.

Godisn, R., \& TheEUwes, J. (2002). Oculomotor capture and inhibition of return. Psychological Research, 66, 234-246.

Hoffman, J. E., \& Mueller, S. (1994, November). An in depth look at visual attention. Paper presented at the annual meeting of the Psychonomic Society, St. Louis.

KLEIN, R. M. (2000). Inhibition of return. Trends in Cognitive Sciences, 4, 138-147.

Klier, E. M., Wang, H., \& Crawford, J. D. (2001). The superior colliculus encodes gaze commands in retinal coordinates. Nature Neuroscience, 4, 627-632.

Lupiáñez, J., Milliken, B., Solano, C., Weaver, B., \& Tipper, S. P. (2001). On the strategic modulation of the time course of facilitation and inhibition of return. Quarterly Journal of Experimental Psychology, 54, 753-773.

Nakayama, K., \& Silverman, G. H. (1986). Serial and parallel processing of visual feature conjunctions. Nature, 320, 264-265.

Posner, M. I. (1980). Orienting of attention. Quarterly Journal of Experimental Psychology, 32, 3-25.

Posner, M. I., \& Cohen, Y. A. (1984). Components of visual orienting. In H. Bouma \& D. G. Bouwhuis (Eds.), Attention and performance X: Control oflanguage processes (pp. 531-556). Hillsdale, NJ: Erlbaum.

Pratt, J., \& ABrams, R. A. (1999). Inhibition of return in discrimination tasks. Journal of Experimental Psychology: Human Perception \& Performance, 25, 229-242.

Pratt, J., Hillis, J., \& Gold, J. M. (2001). The effect of the physical characteristics of cues and targets on facilitation and inhibition. Psychonomic Bulletin \& Review, 8, 489-495.

Rafal, R. D., Calabresi, P. A., Brennan, C. W., \& Sciolto, T. K. (1989). Saccade preparation inhibits reorienting to recently attended locations. Journal of Experimental Psychology: Human Perception \& Performance, 15, 673-685.

Rashbass, C., \& Westheimer, G. (1961). Independence of conjugate and disjunctive eye movements. Journal of Physiology, 159, 361-364.

SPARKS, D. L., \& MAYS, L. E. (1990). Signal transformations required for the generation of saccadic eye movements. Annual Review of Neuroscience, 13, 309-336.

Tassinari, G., \& Berlucchi, G. (1995). Covert orienting to noninformative cues: Reaction time studies. Behavioural Brain Research, 71, 101-102.

TAYLOR, T. L., \& KLEIN, R. M. (1998). On the causes and effects of inhibition of return. Psychonomic Bulletin \& Review, 5, 625-643.

Theeuwes, J. (1991). Exogenous and endogenous control of attention: The effect of visual onsets and offsets. Perception \& Psychopysics, 49, 83-90.

Theeuwes, J., Atchley, P., \& Kramer, A. F. (1998). Attentional control within 3-D space. Journal of Experimental Psychology: Human Perception \& Performance, 24, 1476-1485.

(Manuscript received November 19, 2001; revision accepted for publication May 15, 2002.) 